\title{
Caracterización general de los pacientes con EPOC de la Región del Maule: resultados preliminares del estudio MaulEPOC
}

\author{
JORDI OLLOQUEQUI G.*, SERGIO JAIME J.**, VIVIANA PARRA R.**, CINTIA MUÑOZ V.**, \\ ALEJANDRA MUÑOZ G.***, FABIOLA LASTRA F.***, CRISTIÁN VERGARA E.***, \\ CAROLINA LARA L.***, CÉSAR CAVIEDES O.**, KAREN CZISCHKE L.****, \\ PILAR GARCÍA N.*****, ELIZABETH CORNEJO C.***** y RAFAEL SILVA O.**
}

\section{General characterization of COPD patients from the Maule Region, Chile: Preliminary results of the MaulEPOC study}

This study aimed to assess the epidemiological profile of subjects from the Maule Region (Chile) suffering from Chronic Obstructive Pulmonary Disease (COPD). We recruited 127 stable-COPD patients who were attended by a pulmonologist or treated at Hospital Regional de Talca during 2016. All patients underwent lung functional tests and answered a standardized questionnaire to obtain clinical and epidemiological data. Patients were classified according to the GOLD combined COPD assessment criteria, which included symptomatic assessment with the patient's spirometric classification and risk of exacerbations. GOLD A, B, C and D categories consisted of $25 \%, 33 \%, 9 \%$ and $33 \%$ of patients respectively. The mean age was 71.4 years (CI 95\% 64.7-73.7) and $56 \%$ of the patients were male. A $27 \%$ of subjects completed 4 or less years of schooling. $61 \%$ of patients showed a significant combined exposure to both cigarette and biomass smoke. COPD patients from the Maule Region are mostly elderly male, often showing a low educational level. The main COPD risk factor in this cohort was the simultaneous exposure to cigarette and biomass smoke. Most patients are in the milder stages of COPD. Our findings identified the main areas that can be intervened to improve COPD management in the Maule Region.

Key words: Pulmonary disease, chronic obstructive; risk factors; smoke; biomass; surveys and questionnaires.

\section{Resumen}

El objetivo de esta comunicación fue evaluar el perfil epidemiológico de los pacientes con Enfermedad Pulmonar Obstructiva Crónica (EPOC) de la Región del Maule. Para ello, se contó con 127 pacientes EPOC clínicamente estables derivados por parte del neumólogo o que fueron atendidos en el centro de diagnóstico terapéutico del Hospital Regional de Talca durante el año 2016. Los pacientes fueron sometidos a una serie de pruebas de función pulmonar y debieron contestar un cuestionario para conocer antecedentes clínicos y epidemiológicos. Tras el estudio, fueron clasificados de acuerdo al criterio GOLD que integra medidas de síntomas, disnea, espirometría y riesgo de exacerbaciones. El $56 \%$ de los pacientes fueron varones y el promedio de edad en ambos sexos fue de 71,4 años (IC 95\% $67,89-71,28)$. Un 27\% tuvieron un nivel de escolarización $\leq 4$ años. El $25 \%$ fueron clasificados como

Fuente de financiamiento: Este trabajo ha sido financiado por el Fondo Nacional de Desarrollo Científico y Tecnológico (FONDECYT \#11150022) de CONICYT.

\footnotetext{
* Instituto de Ciencias Biomédicas, Facultad de Ciencias de la Salud, Universidad Autónoma de Chile. Talca, Chile.

** Unidad Respiratorio, Centro de Diagnóstico Terapéutico, Hospital Regional de Talca, Talca, Chile.

*** Carrera de Enfermería, Facultad de Ciencias de la Salud, Universidad Autónoma de Chile. Talca, Chile.

**** Hospital Padre Hurtado, Santiago, Chile.

*****Subdepartamento de Salud Laboral, Hospital Regional de Talca, Talca, Chile.
} 
GOLD A, el 33\% B, el 9\% Cy el 33\% D. El 61\% de los pacientes presentaron una exposición significativa simultánea a humo de cigarrillo y humo de biomasa. Los pacientes EPOC de la Región del Maule son fundamentalmente varones, adultos mayores y, frecuentemente, con bajo nivel de escolaridad. Los principales factores de riesgo para el desarrollo de la enfermedad en esta cohorte fueron la exposición simultánea a humo de tabaco y de combustibles de biomasa. La mayoría de los pacientes se encuentran en los estadios más leves de la enfermedad. Nuestros hallazgos identificaron las principales áreas que deben ser intervenidas para mejorar el manejo de la EPOC en la Región del Maule.

Palabras clave: EPOC; factores de riesgo; humo; biomasa; encuestas y cuestionarios.

\section{Introducción}

La enfermedad pulmonar obstructiva crónica (EPOC) se caracteriza por una limitación al flujo aéreo asociada a una respuesta inflamatoria anormal de los pulmones ante determinadas partículas o gases ${ }^{1}$. Aunque el humo del cigarrillo es el principal factor de riesgo para desarrollar la enfermedad, existe cada vez más evidencia que la exposición intradomiciliaria al humo de combustibles de biomasa es también un importante elemento susceptible de causar EPOC ${ }^{2}$.

La EPOC se ha convertido en una de las principales causas de morbimortalidad a nivel global, con elevadas cargas sociales y económicas ${ }^{3}$. A pesar de que su prevalencia varía en función del país, se estima que 35 de cada 1.000 hospitalizaciones en América Latina se deben a la EPOC, con rangos de mortalidad intrahospitalaria que van del $6,7 \%$ al $29,5 \%{ }^{4}$. Además, se espera que la prevalencia de ésta y otras enfermedades asociadas al adulto siga incrementándose en los países latinoamericanos, que prácticamente han cuadriplicado su población desde el año 1950 y que han aumentado significativamente su esperanza de vida en las últimas décadas ${ }^{5}$.

Según un estudio descriptivo realizado el año 1999, las enfermedades respiratorias constituyen la tercera causa de muerte en Chile y, entre ellas, la EPOC ocupa el segundo lugar ${ }^{6}$. Con todo, la información acerca de la prevalencia de esta patología en América Latina en general $y$ en Chile en particular fue muy escasa hasta la presentación en el año 2005 de los resultados del Proyecto Latinoamericano de Investigación en Obstrucción Pulmonar (PLATINO), el cual reportó que el $16,9 \%$ de la población mayor de 40 años en Santiago de Chile sufría EPOC ${ }^{7}$. A pesar del punto de inflexión que supuso el proyecto PLATINO para comprender la realidad de la EPOC en Chile, dicho estudio solo consideró a la ciudad de Santiago, por lo que la información acerca del perfil epidemiológico de los pacientes EPOC de otras regiones chilenas sigue siendo prácticamente inexistente. Además, desde el año 2011 la Iniciativa Global para la Enfermedad Pulmonar Obstructiva Crónica (GOLD) recomendó el uso de una herramienta de evaluación combinada con datos de síntomas, limitación del flujo aéreo y exacerbaciones para guiar la terapia de los pacientes con EPOC.

Teniendo en cuenta los antecedentes mencionados, el proyecto MaulEPOC nació con el objetivo de llevar a cabo una caracterización demográfica, clínica y molecular de los pacientes con EPOC de la Región del Maule. El presente trabajo recoge una caracterización preliminar de los pacientes EPOC maulinos reclutados en el 2016 y evaluados según el criterio combinado de la GOLD.

\section{Método}

\section{Población de estudio}

Los sujetos de estudio fueron mayormente pacientes derivados por un neumólogo del Hospital Regional de Talca (HRT) entre los meses de marzo y noviembre del 2016 para la realización de pruebas funcionales por sospecha de EPOC. Además, también se reclutaron otros pacientes ya atendidos por EPOC en el centro de diagnóstico terapéutico (CDT) del HRT y que acudieron para control durante ese período. Todos ellos firmaron un consentimiento informado, aprobado por el Comité de Ética del Servicio de Salud del Maule y el Comité de Ética de la Universidad Autónoma de Chile.

Tras la confirmación diagnóstica, los pacientes EPOC clínicamente estables (sin exacerbaciones durante los dos últimos meses) fueron citados en la unidad cardiorrespiratoria del CDT para la realización de pruebas funcionales adicionales. Además, se les tomó una muestra de sangre para posteriores análisis moleculares y contestaron un cuestionario estandarizado para recolectar antecedentes biodemográficos y clínicos. Sólo se incluyeron en el estudio aquellos pacientes de los que se pudieron obtener todos los datos. 


\section{Características clínicas y biodemográficas}

El diagnóstico de EPOC se confirmó de acuerdo a la definición propuesta por la GOLD, que consiste en una razón entre el volumen espiratorio forzado en el primer segundo $\left(\mathrm{VEF}_{1}\right)$ y la capacidad vital forzada (CVF) inferior a 0,7 posterior al uso de broncodilatador (relación fija) ${ }^{1}$. Las espirometrías fueron realizadas por personal previamente entrenado utilizando un espirómetro modelo CPFS/D (MGC Diagnostics, Alaska, Estados Unidos). Se realizó un mínimo de 3 maniobras y un máximo de 8 , dejando entre ellas el tiempo suficiente para que el paciente se recuperara del esfuerzo. Para efectos de normativa del Ministerio de Salud de Chile, debieron existir al menos 3 curvas similares, con una diferencia inferior a $0,15 \mathrm{~L}$. Finalmente, se escogió aquella curva que tuviera los valores más altos para realizar las comparaciones con la espirometría post broncodilatador. Ésta se realizó con el objetivo de efectuar el diagnóstico diferencial entre la EPOC y el asma bronquial. Para ello, el paciente inhaló 4 puffs de Salbutamol $(400 \mu \mathrm{g}$ en total). En caso de presentar una historia de reacciones adversas a dicho fármaco, tales como la taquicardia, se prefirió inhalar con 8 puffs de Bromuro de Ipatropio (160 $\mu \mathrm{g}$ en total). Una vez realizada la inhalación con cualquiera de los broncodilatadores antes descritos, se procedió a esperar un mínimo de 10 min. Pasado el tiempo de espera, se realizó nuevamente la espirometría. Finalmente, se seleccionó la curva con los valores más altos para establecer comparación con la espirometría basal; se consideró que una respuesta broncodilatadora era significativa si presentaba un cambio igual o mayor a $12 \%$ y 200 $\mathrm{mL}$ en el $\mathrm{VEF}_{1}$ y/o CVF respecto al valor basal, pre-broncodilatador. Si la espirometría basal mostraba una obstrucción al flujo aéreo y el paciente presentaba una respuesta broncodilatadora positiva, este era catalogado como asmático y no se incluyó en el estudio.

Para la realización del test de difusión con monóxido de carbono (DLCO) se dispuso de una fuente de gas comprimido (Indura, Talca, Chile) compuesta por monóxido de carbono $(0,3 \%)$, gas metano $(0-3 \%)$, oxígeno $(21 \%)$ y nitrógeno conectada a un computador de escritorio que incluía el programa para pletismografía Vmax Program Manager (SensorMedic, Florida, Estados Unidos). La fuente de gas y el computador se unen mediante válvulas con el pletismógrafo.

Para el test de caminata en 6 min se utilizó un esfigmomanómetro Dinamap (Critikon, Florida, Estados Unidos) para la medición de la presión arterial y la frecuencia cardiaca. Se utilizó también un oxímetro de pulso marca Ohmeda TuffSat (Soma Technology, Connecticut, Estados Unidos) para medir la saturación de oxígeno de la hemoglobina. Por otra parte, se usó un tallímetro/balanza Health-o-meter (Sunbeam, Florida, Estados Unidos), para medir datos antropométricos como el peso y talla. Además, se requirió de un cronómetro con el objetivo de medir el tiempo del recorrido.

Para la caracterización biodemográfica y clínica de los sujetos se recabó información sobre antecedentes sociales y demográficos, tratamiento actual, comorbilidades, índice $\mathrm{CAT}^{8}$, grado de disnea según la escala modificada del Medical Research Council (mMRC) ${ }^{9}$, número de exacerbaciones en los últimos dos años y en cuántas de ellas se requirió hospitalización, entre otras variables. También se registró antecedentes de exposición a factores de riesgo de EPOC conocidos, como el historial tabáquico (índice paquetes-año-IPA), exposición intradomiciliaria a humo de biomasa y exposiciones ocupacionales. Se consideraron personas expuestas a humo de biomasa aquellas que refirieron el uso de estufa/chimenea/cocina en su hogar mediante combustión de madera, coque, pellet, carbón, restos vegetales o estiércol durante más de 6 meses en su vida. La exposición acumulada a humo de biomasa (horas-año) se calculó mediante el cociente del número de años cocinando o calefaccionándose con biomasa multiplicado por la media aproximada de horas que se pasan en la cocina/cerca de la fuente de calefacción ${ }^{10}$. Se consideró como personas con exposición significativa a polvo de origen ocupacional a aquellas que trabajaron durante 3 meses o más en un ambiente con partículas o humo ${ }^{11}$. Finalmente, los sujetos fueron clasificados de acuerdo al instrumento de evaluación "ABCD" perfeccionado de la GOLD ${ }^{1}$ que integra los datos de la espirometría, los síntomas del paciente y los antecedentes de exacerbaciones.

\section{Análisis estadístico}

Se realizó un análisis descriptivo para las variables categóricas, las cuales se expresaron en número de sujetos y porcentaje. Las variables continuas fueron expresadas con la media y desviación estándar. Para estas últimas, se realizó un análisis de la varianza ANOVA, tomando como significativo un $p$ valor inferior a 0,05 . Todos los análisis se llevaron a cabo con el paquete estadístico R (R: A language and environment for statistical computing. $R$ Foundation for Statistical Computing, Viena, Austria). 


\section{Resultados}

\section{Características sociodemográficas y categorías GOLD}

La Tabla 1 muestra un resumen de las características sociodemográficas y categorías GOLD de los sujetos de estudio. Se logró reclutar y caracterizar a un total de 127 pacientes. Aproximadamente un $25 \%$ de los casos presentó severidad GOLD A, un 32\% severidad GOLD B, un 34\% severidad GOLD D y tan sólo un $9 \%$ presentó severidad GOLD C, siendo el grupo minoritario (Tabla 1).

Respecto a la distribución del grupo de pacientes según sexo, nuestra cohorte estuvo formada por 71 pacientes varones, lo que representa un $56 \%$ del total. Por otro lado, el número de mujeres reclutadas fue de 56 , lo que representa el $44 \%$ del total. Los grupos GOLD A y D presentaron una mayoría de varones ( 72 y $56 \%$, respectivamente) mientras que los grupos B y D estuvieron formados por más mujeres que hombres $(54 \mathrm{y}$ $46 \%$, respectivamente).

El promedio de edad de todos los pacientes EPOC fue de 71,40 años (IC 95\% 67,89-71,28) y no existieron diferencias significativas entre los cuatro grupos GOLD. En cuanto al nivel de escolarización, la mayoría declaró haber asistido a algún centro educacional durante alguna etapa de su vida. Sin embargo, un alto porcentaje de pacientes de nuestra cohorte $(27 \%)$ tuvo un nivel de escolaridad igual o menor a 4 años. Por otra parte, el $31 \%$ de los pacientes declaró tener un nivel de escolaridad entre 5 y 8 años, mientras que el $24 \%$ refirió tener un nivel de escolaridad de entre 9 a 12 años. Solo el 7\% de los sujetos manifestó un nivel de escolaridad mayor o igual a 13 años. Finalmente, el $11 \%$ de los pacientes refirió no haber asistido nunca al colegio. Aunque el grupo D mostró el menor promedio de años de escolarización completados, no existieron diferencias significativas entre los cuatro grupos GOLD para esta variable (Tabla 1).

\section{Características clínicas}

En la Tabla 2 se muestran las principales características clínicas de los pacientes agrupados según el criterio integrado GOLD. El promedio del índice de masa corporal (IMC) para la cohorte fue de 27,45 (CI 95\% 26,38-28,52). No existieron diferencias significativas entre grupos en cuanto al IMC, cuyos promedios se situaron por encima de 25 y por debajo de 30 en todos los grupos, poniendo de manifiesto un sobrepeso generalizado en los enfermos de EPOC maulinos. Por el contrario, el valor del $\mathrm{VEF}_{1}$ disminuyó desde el grupo A hasta el $\mathrm{D}$, habiendo diferencias significativas entre los cuatro grupos de estudio. Tanto el cociente $\mathrm{VEF}_{1} / \mathrm{CVF}$ como la saturación de oxigeno de la hemoglobina fueron significativamente menores en los grupos más graves $(\mathrm{C}$ y $\mathrm{D})$ respecto a los más leves $(\mathrm{A}$ y $\mathrm{B})$. El valor de la capacidad de difusión ( $\left(\mathrm{DL}_{\mathrm{CO}}\right)$ fue significativamente inferior en los pacientes del grupo $\mathrm{D}$ en comparación a los de los grupos A y B. Finalmente, los pacientes del grupo $\mathrm{D}$ presentaron un grado de disnea significativamente mayor en comparación a los otros grupos, así como resultados significativamente peores en los tests de caminata y de la calidad de vida en comparación a los grupos A y B (Tabla 2).

\section{Exposición a factores de riesgo}

E1 90\% de los pacientes reclutados declararon haber sido fumadores alguna vez, aunque sólo el $7 \%$ seguía con el hábito. Por otra parte, el 71\% de los pacientes reportó haber estado expuesto a contaminación intradomiciliaria por humo de combustibles de biomasa durante más de 6 meses en su vida. En cuanto al porcentaje de los pacientes con exposición tanto al humo del tabaco como al de la biomasa, este fue del $61 \%$. El porcentaje de sujetos que refirieron haber estado expuestos a polvos ocupacionales fue del $56 \%$.

Los datos acerca de la exposición a factores de riesgo según grupos GOLD se presentan en la Tabla 3. Los grupos $\mathrm{B}$ y $\mathrm{C}$ presentaron un

Tabla 1. Características Sociodemográficas de 127 pacientes con EPOC de la Región del Maule

\begin{tabular}{|lcccc|}
\hline & Grupo A & Grupo B & Grupo C & Grupo D \\
\hline Población muestral, n (\%) & $32(25,19)$ & $41(32,28)$ & $11(8,66)$ & $43(33,85)$ \\
Género & & & & \\
Masculino, n (\%) & $23(71,87)$ & $19(43,34)$ & $4(44,44)$ & $24(55,81)$ \\
Femenino, n (\%) & $9(28,12)$ & $22(53,65)$ & $5(45,45)$ & $19(44,18)$ \\
Edad, años* & $68,31 \pm 8,36$ & $70,85 \pm 9,56$ & $69,72 \pm 7,21$ & $70,47 \pm 8,41$ \\
Escolarización, años completados & $7,13 \pm 4,59$ & $7,71 \pm 4,83$ & $6,00 \pm 4,63$ & $5,63 \pm 3,83$ \\
\hline
\end{tabular}

*Edad en años: Valores expresados con la media \pm desviación estándar. 
Tabla 2. Características Clínicas de 127 pacientes con EPOC de la región del Maule agrupados según GOLD

\begin{tabular}{|lcccc|}
\hline & Grupo A & Grupo B & Grupo C & Grupo D \\
\hline $\mathrm{IMC}, \mathrm{kg} / \mathrm{m}^{2}$ & $28,9 \pm 4,48$ & $27,45 \pm 4,63$ & $29,27 \pm 6,15$ & $26,27 \pm 4,49$ \\
\hline $\mathrm{VEF}_{1}, \%$ del predicho & $75,47 \pm 22,29$ & $68,07 \pm 9,93^{\mathrm{a}}$ & $41,81 \pm 8,29^{\mathrm{a}, \mathrm{b}}$ & $29,93 \pm 9,35^{\mathrm{a}, \mathrm{b}, \mathrm{c}}$ \\
\hline $\mathrm{VEF}_{1} / \mathrm{CVF}, \%$ & $60,44 \pm 7,15$ & $61,75 \pm 5,17$ & $53,09 \pm 10,31^{\mathrm{a}, \mathrm{b}}$ & $46,00 \pm 10,57^{\mathrm{a}, \mathrm{b}, \mathrm{c}}$ \\
$\mathrm{DL}_{\mathrm{cO}}, \%$ del predicho & $14,23 \pm 18,74$ & $12,58 \pm 19,97$ & $11,51 \pm 19,24$ & $9,39 \pm 11,21^{\mathrm{a}, \mathrm{b}}$ \\
\hline Oximetría,$\%$ & $94,08 \pm 3,51$ & $94,00 \pm 4,21$ & $89,75 \pm 5,44^{\mathrm{a}, \mathrm{b}}$ & $89,93 \pm 3,79^{\mathrm{a}, \mathrm{b}}$ \\
\hline Caminata 6 min, m & $445,50 \pm 106,89$ & $365,96 \pm 139,04$ & $322,13 \pm 131,84^{\mathrm{a}}$ & $271,19 \pm 164,74^{\mathrm{a}, \mathrm{b}}$ \\
mMRC & $1,30 \pm 0,75$ & $2,73 \pm 1,14^{\mathrm{a}}$ & $2,20 \pm 0,63^{\mathrm{a}}$ & $3,29 \pm 0,74^{\mathrm{a}, \mathrm{b}, \mathrm{c}}$ \\
\hline CAT & $5,97 \pm 2,64$ & $14,35 \pm 2,55^{\mathrm{a}}$ & $15,57 \pm 7,64^{\mathrm{a}}$ & $17,23 \pm 5,19^{\mathrm{a}, \mathrm{b}}$ \\
\hline
\end{tabular}

Valores expresados con la media \pm desviación estándar. ${ }^{a}$ Indica una diferencia significativa respecto a $\mathrm{A}(\mathrm{p}<0,05)$. bIndica una diferencia significativa respecto a $B(p<0,05)$. ' Indica una diferencia significativa respecto a $C(p<0,05)$. $\mathrm{VEF}_{1}$ : volumen espiratorio forzado en el primer segundo; CVF: capacidad vital forzada; $\mathrm{DL}_{\mathrm{co}}$; capacidad de difusión del monóxido de carbono; C6M: test de caminata en 6 min; mMRC: escala de disnea modificada del Medical Research Council; CAT: Test del calidad de vida "COPD Assessment Test".

Tabla 3. Exposición a factores de riesgo

\begin{tabular}{|lccrr|}
\hline & Grupo A & Grupo B & Grupo C & Grupo D \\
Estatus tabáquico & & & & \\
$\quad$ Nunca fumó, n (\%) & $3(9,37)$ & $6(14,63)$ & $3(27,27)$ & $4(9,30)$ \\
Fumador actual, n (\%) & $4(12,5)$ & $5(12,19)$ & $1(9,09)$ & $5(11,63)$ \\
$\quad$ Exfumador, n (\%) & $25(78,12)$ & $30(73,17)$ & $7(77,77)$ & $34(79,06)$ \\
Índice paquete-año & $40,98 \pm 3,86$ & $28,71 \pm 28,49$ & $24,27 \pm 24,61$ & $42,34 \pm 34,68$ \\
Exposición a humo de biomasa, n (\%) & $27(84,3)$ & $36(87,80)$ & $8(88,88)$ & $38(88,37)$ \\
Exposición a biomasa, horas-año & $130,32 \pm 124,87$ & $217,45 \pm 193,98$ & $174,90 \pm 113,95$ & $261,37 \pm 248,77$ \\
Exposición ocupacional, n (\%) & $22(68,75)$ & $18(43,90)$ & $1(11,11)$ & $25(58,13)$ \\
\hline
\end{tabular}

Valores expresados como número de sujetos (porcentaje) o con la media \pm desviación estándar.

mayor índice de paquetes-año, a pesar de que las diferencias entre los 4 grupos no fueron significativas. En cuanto a la exposición acumulada a humo de biomasa, el grupo D presentó un valor sensiblemente más elevado, aunque tampoco hubo diferencias estadísticamente significativas.

\section{Comorbilidades asociadas}

Un $78 \%$ de los pacientes refirieron padecer comorbilidades asociadas, siendo la hipertensión arterial la más común ( $72 \%$ de los pacientes con comorbilidades). Un $7 \%$ presentaba diabetes mellitus y el $17 \%$ refirió infección previa de tuberculosis.

\section{Historial de exacerbaciones}

El porcentaje de sujetos que había presentado al menos una exacerbación durante el año anterior fue de un $48,03 \%$, frente al $51,96 \%$ de sujetos que no presentaron exacerbaciones en ese período. En la Figura 1 se muestran las cifras de pacientes con exacerbaciones ambulatorias y hospitalizadas. Sólo un $21,31 \%$ de las exacerbaciones requirieron de hospitalización. La mayor parte de los pacientes $(57,38 \%)$ presentaron dos o más exacerbaciones de carácter ambulatorio.

\section{Tratamiento farmacológico}

Casi la totalidad de los pacientes $(93,7 \%)$ estaban bajo tratamiento para EPOC al momento en que se les realizó el cuestionario. Entre ellos, el $14,29 \%$ seguían terapia con un solo fármaco broncodilatador $\beta$-adrenérgico o anticolinérgico de acción corta. El resto $(85,71 \%)$ seguían una terapia compuesta por corticoides inhalados con uno o dos broncodilatadores de acción larga. Cabe destacar que el uso de corticoides inhalados se distribuyó de forma homogénea entre los cuatro grupos GOLD. La terapia mayoritaria, con el $80,67 \%$ de los pacientes bajo tratamiento, 


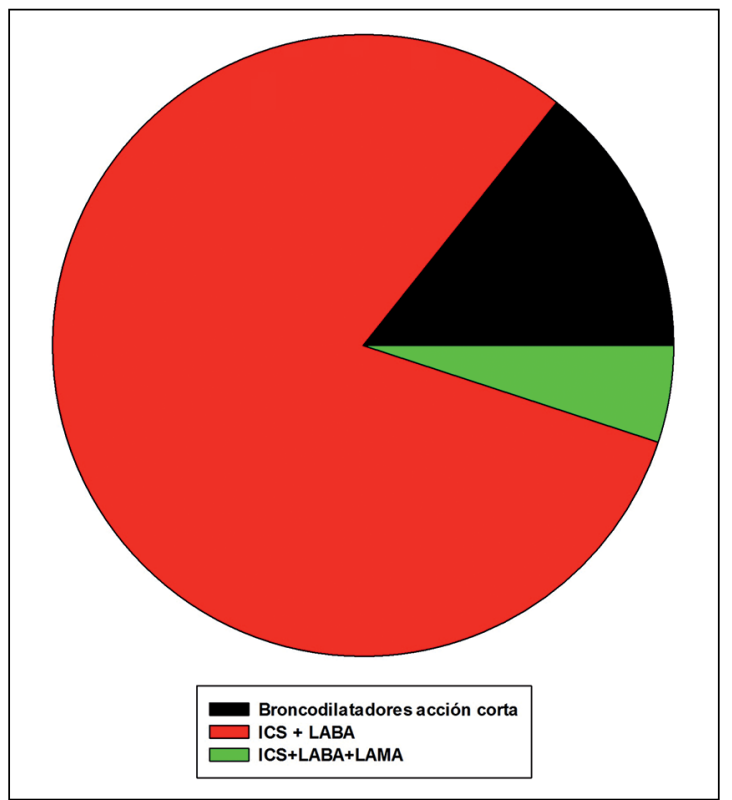

Figura 1. Exacerbaciones ambulatorias y hospitalizadas. Aproximadamente el $48 \%$ de los pacientes $(n=61)$ presentaron alguna exacerbación durante el último año. Sólo 13 pacientes tuvieron que ser hospitalizados por alguna de estas exacerbaciones. La mayoría de los pacientes $(\mathrm{n}=35)$ presentaron dos o más exacerbaciones de carácter ambulatorio.

fue de corticoides inhalados más $\beta$-adrenérgicos de acción prolongada (LABA). Por último, un $5,04 \%$ de los pacientes bajo tratamiento siguieron una triterapia formada por corticoides inhalados + LABA + anticolinérgicos de acción prolongada (LAMA, Figura 2).

\section{Comentario}

La EPOC es una enfermedad muy prevalente a nivel global y se espera que siga en aumento durante las próximas décadas, probablemente por la alta tasa de exposición a sus principales factores de riesgo ambientales ${ }^{12}$, entre otras causas. En este sentido, el panorama en nuestro país no parece muy diferente al de las estimaciones globales. De acuerdo a la Organización Panamericana de la Salud, Chile encabeza la lista de países sudamericanos con el número más alto de fumadores jóvenes, con un porcentaje de adolescentes entre 15 y 18 años fumadores del $32,9 \%{ }^{13}$. Por otro lado, se estima que entre el 30 y el $75 \%$ de hogares en zonas rurales de América Latina usan habitualmente la biomasa como combustible, para cocinar o para calefacción ${ }^{14}$.

Hasta donde conocemos, el presente estudio es el primero en establecer una caracterización general de los pacientes EPOC de la Región del Maule, una zona muy poco estudiada desde el punto de vista de la investigación biomédica sobre EPOC y otras enfermedades respiratorias, pero con un alto interés por varias razones. En primer lugar, porque presenta una tasa de tabaquismo del $22,6 \%$, situándose entre la seis regiones con mayor tabaquismo del país ${ }^{15}$. En segundo lugar, porque la Región del Maule presenta un $32,9 \%$ de población rural, la cifra más alta de Chile $^{16}$, con lo cual, es esperable una alta tasa de exposición al humo de biomasa. Además, la prevalencia de síntomas respiratorios crónicos en la Región del Maule, evaluada

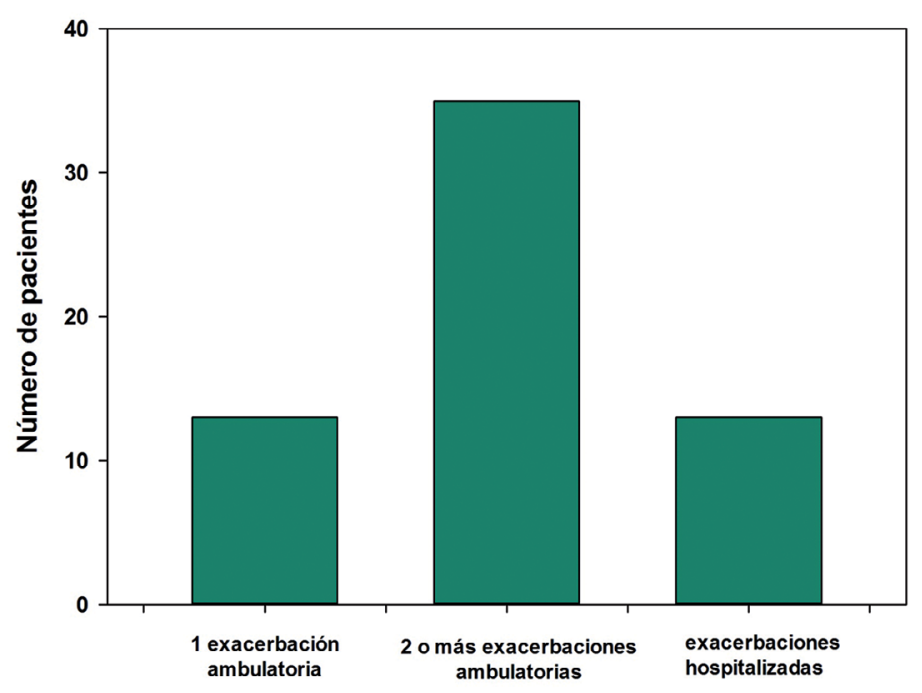

Figura 2. Tratamientos farmacológicos más frecuentes. La mayoría de los pacientes bajo tratamiento $(85,71 \%)$ incluyeron el uso de corticoides inhalados. El tratamiento mayoritario en la cohorte estudiada fue el compuesto por corticoides inhalados y $\beta$-adrenérgicos de acción prolongada. 
en la Encuesta Nacional de Salud 2009-2010 fue del $31,5 \%$, cifra similar a la observada en el resto del país.

La mayoría de estudios a nivel mundial muestran una mayor prevalencia de EPOC en hombres que en mujeres y en personas de edades avanzadas $^{1}$. Estos datos coinciden con los de nuestra cohorte, en la que predominaron los pacientes varones y con un promedio de edad de 71 años para ambos sexos. Un elevado porcentaje de los pacientes $(27 \%)$ refirió un nivel de escolaridad igual o inferior a 4 años y la mayoría de ellos se encontraban en los estadios GOLD A y B. Estos datos son relevantes y sugieren que podría existir una alta tasa de subdiagnóstico de EPOC en la Región del Maule, puesto que se ha reportado que esta enfermedad se subdiagnostica especialmente en pacientes jóvenes, en aquellos que se encuentran en los estadios más leves y en personas con bajo nivel educacional ${ }^{17}$.

La caracterización clínica de nuestra cohorte puso de manifiesto un sobrepeso generalizado (IMC entre 25 y $29,9 \mathrm{~kg} / \mathrm{m}^{2}$ ) tanto en el promedio total como en el de cada grupo GOLD. Esto coincide con los resultados de otros estudios internacionales ${ }^{18}$, así como también los obtenidos en la cohorte chilena del estudio PLATINO ${ }^{19}$ y en una cohorte de sujetos EPOC de la comuna de Puente Alto, Santiago ${ }^{20}$. Como era de esperar, los pacientes clasificados como GOLD D mostraron los peores valores espirométricos, de $\mathrm{DL}_{\mathrm{CO}}$, de saturación de oxígeno de la hemoglobina, del test de caminata y de calidad de vida, mostrando diferencias significativas con los grupos A y B. Además, este grupo mostró un incremento estadísticamente significativo de la disnea en comparación a los otros tres. Estos resultados respaldan el valor diagnóstico del criterio integrado de la GOLD en nuestra cohorte.

En cuanto a los factores de riesgo principales, la inmensa mayoría de los pacientes habían sido fumadores, sin observarse diferencias significativas en el IPA entre los cuatro grupos GOLD. No obstante, el Grupo D fue el que presentó un IPA más alto, curiosamente seguido por el Grupo A. El hecho de que los pacientes del Grupo A hubieran fumado más que los de los dos estadios siguientes refleja la naturaleza multifactorial de la EPOC ${ }^{21}$. En relación a la exposición de humo de biomasa intramuros, ésta fue reportada por más de dos tercios de la cohorte maulina. En este caso, los grupos B y D mostraron una mayor exposición acumulada a este contaminante. Tales resultados contrastan con los de la cohorte chilena del estudio PLATINO, donde la exposición intradomiciliaria a humo de biomasa fluctuó entre el 55,2\% para carbón y el 55,8\% para la leña ${ }^{22}$. Probablemente, el hecho de que la prevalencia de la exposición a humo de biomasa sea más alta en nuestra cohorte refleja el alto porcentaje de población rural en la Región del Maule, más proclive al uso de este tipo de combustibles. Por otra parte, la exposición combinada tanto al humo del cigarrillo como al de biomasa fue muy alta en nuestra cohorte, coincidiendo con lo reportado en la cohorte de Puente $\mathrm{Alto}^{20}$. Otro factor de riesgo importante para desarrollar la EPOC en esta región fue la exposición ocupacional. La mayoría de los pacientes afirmó haber trabajado en lugares donde hubo exposición a sustancias como harinas, polvos y gases de fabricación de plásticos, etc. Este dato es relevante, puesto que estimaciones basadas en datos internacionales, entre los que se hallaban los del estudio PLATINO, señalan que podría reducirse un $20 \%$ de la carga que supone la EPOC mediante la reducción de un $8,8 \%$ de la prevalencia de las exposiciones ocupacionales $^{23}$. A pesar de que las condiciones a las que son sometidos los trabajadores chilenos en la actualidad han mejorado, se debe tener en cuenta que casi la totalidad de los sujetos de nuestra cohorte fueron adultos mayores jubilados. En consecuencia, durante la época en que se encontraban en edad activa, probablemente sus condiciones laborales fueron más precarias y las leyes no les garantizaron una protección o prevención adecuadas ante enfermedades ocupacionales ${ }^{24}$.

Una gran mayoría de nuestra cohorte (78\%) presentó comorbilidades asociadas, siendo la hipertensión arterial la más común. Esto coincide con los resultados de otros estudios tanto nacionales como internacionales ${ }^{20,25,26}$ y es de suma importancia para el seguimiento de los pacientes, dado que las comorbilidades merman la calidad de vida de los enfermos de EPOC y son responsables de su mortalidad en muchos $\operatorname{casos}^{27}$. En cuanto al historial de exacerbaciones, casi la mitad de nuestra cohorte manifestó haber padecido alguna exacerbación durante el último año $(48 \%)$. Esta cifra es muy superior a la de los resultados globales del proyecto PLATINO, reportando que sólo un 7,8\% de su cohorte con EPOC había presentado alguna exacerbación en el último año ${ }^{28}$. Esto pone de manifiesto una necesidad urgente de programas específicos de información y prevención de exacerbaciones en los enfermos de EPOC maulinos.

En referencia al tratamiento farmacológico, nuestra cohorte se encuentra mayormente bajo terapias en la que se combinan uno o más bron- 
codilatadores con corticoides inhalados. Estos resultados contrastan con los de la cohorte general del estudio PLATINO ${ }^{29}$, para la que se reportó el uso de corticoides inhalados en sólo el 13,5\% de los sujetos con EPOC más grave. Puesto que los corticoides presentan importantes efectos adversos y sus ventajas en el tratamiento de la EPOC (ya sea de forma simple o combinada) siguen siendo polémicas ${ }^{30}$, creemos que el uso frecuente de corticoides inhalados es un tema que requiere de un análisis profundo por las implicaciones que puede tener sobre la población EPOC maulina. Por otro lado, el bajo uso de la terapia LAMA-LABA en nuestra serie se explica por no estar disponible esta terapia en el Hospital Regional durante en la fecha de reclutamiento de pacientes.

El presente trabajo preliminar tiene varias limitaciones. En primer lugar, la cantidad de pacientes reclutados no fue muy alta durante el período estudiado y eso ha impedido contar con un número balanceado de casos en los cuatro grupos GOLD. Por otra parte, hasta la fecha no hemos tenido acceso a resultados imagenológicos de la cohorte, que nos podrían haber aportado valiosa información sobre el fenotipo predominante (bronquítico o enfisematoso) de los pacientes EPOC maulinos. En cuanto a la caracterización molecular prevista en el proyecto MaulEPOC, todavía no tenemos datos concluyentes al no haber alcanzado la población muestral total. A diferencia del estudio PLATINO, de base poblacional, en el presente trabajo sólo se consideraron sujetos derivados en base a su condición de EPOC. No obstante, estos resultados preliminares han posibilitado caracterizar por primera vez y de manera general a los enfermos EPOC del Maule, lo que permite tener una visión amplia de cómo la enfermedad está impactando a nivel local.

En definitiva, el presente estudio muestra que los pacientes EPOC maulinos son fundamentalmente varones, adultos mayores y con una tasa importante de baja escolarización. La mayoría presenta sobrepeso y frecuentemente hipertensión. No obstante, hay un predominio de casos que se encuentran en los estadios más leves de la enfermedad. La cohorte muestra una alta prevalencia de exposición combinada a humo de tabaco y de biomasa, siendo también importante la tasa de exposición a factores de riesgo ocupacionales. Nuestro estudio ha puesto de manifiesto las variables principales que deberían tomarse en cuenta para establecer mejoras en la prevención, tratamiento y monitorización de los enfermos de EPOC de la región.

\section{$\underline{\text { Agradecimientos }}$}

Los autores agradecen a la Srta. Jeannete Villegas Arellano, la Sra. Verónica Figueroa Oyarce y a la Srta. Hanuxa Celedón González el apoyo técnico prestado en la investigación. También agradecen al Dr. Gonzalo Valdivia Cabrera su aporte intelectual durante la gestación del proyecto MaulEPOC.

\section{Bibliografía}

1.- GLOBAL INITIATIVE FOR CHRONIC LUNG DISEASES (GOLD). Global Strategy for the Diagnosis, Management and Prevention of COPD-2016. Disponible en: http://www.goldcopd.org.

2.- SILVA R, OYARZÚN M, OLLOQUEQUI J. Pathogenic mechanisms in chronic obstructive pulmonary disease due to biomass smoke exposure. Arch Bronconeumol 2015; 51: 285-92.

3.- LOPEZ-CAMPOS J L, TAN W, SORIANO J B. Global burden of COPD. Respirology 2016; 21: 14-23.

4.- CIAPPONI A, ALISON L, AGUSTINA M, DEMIAN G, SILVANA C, EDGARDO S. The epidemiology and burden of COPD in Latin America and the Caribbean: systematic review and meta-analysis. Copd 2014; 11 : 339-50.

5.- SOARES R R. Life expectancy and welfare in Latin America and the Caribbean. Health Econ 2009; 18 Suppl 1: S37-54.

6.- SZOT M J. Mortalidad por enfermedades respiratorias en Chile durante 1999. Rev Chil Enferm Respir 2003; 19: 8-14.

7.- MENEZES A M B, PÉREZ-PADILLA R, JARDIM JB, MUIÑO A, LÓPEZ MV, VALDIVIA G, et al. Chronic obstructive pulmonary disease in five Latin American cities (the PLATINO study): a prevalence study. Lancet; 366 (9500): 1875-81.

8.- JONES P W, HARDING G, BERRY P, WIKLUND I, CHEN W H, KLINE LEIDY N. Development and first validation of the COPD Assessment Test. Eur Respir J 2009; 34: 648-54.

9.- CELLI B R, COTE C G, MARÍN J M, CASANOVA C, MONTES DE OCA M, MÉNDEZ R A, et al. The Body-Mass Index, Airflow Obstruction, Dyspnea, and Exercise Capacity Index in Chronic Obstructive Pulmonary Disease. New Engl J Med 2004; 350: 1005-12.

10.- RAMÍREZ-VENEGAS A, SANSORES RH, PÉREZPADILLA R, REGALADO J, VELÁZQUEZ A, SÁNCHEZ C, et al. Survival of patients with chronic obstructive pulmonary disease due to biomass smoke and tobacco. Am J Respir Crit Care Med 2006; 173: 393-7.

11.- TAN WC, SIN DD, BOURBEAU J, HERNÁNDEZ P, CHAPMAN KR, COWIE R, et al. Characteristics 
of COPD in never-smokers and ever-smokers in the general population: results from the Can COLD study. Thorax 2015; 70: 822-9.

12.- ADELOYE D, CHUA S, LEE C, BASQUILL C, PAPANA A, THEODORATOU E, et al. Global and regional estimates of COPD prevalence: Systematic review and meta-analysis. J Glob Health 2015; 5: 020415. doi: 10.7189/jogh.05-020415.

13.- MÜLLER F, WEHBE L. Smoking and smoking cessation in Latin America: a review of the current situation and available treatments. Int J Chron Obstruct Pulmon Dis 2008; 3: 285-93.

14.- TORRES-DUQUE C, MALDONADO D, PÉREZPADILLA R, EZZATI M, VIEGI G. Biomass fuels and respiratory diseases: a review of the evidence. Proc Am Thorac Soc 2008; 5: 577-90.

15.- MINISTERIO DE DESARROLLO SOCIAL. Informe de desarrollo social. 2012. Disponible en: http://www. ministeriodesarrollosocial.gob.cl/pdf/upload/IDS2012. pdf.

16.- INSTITUTO NACIONAL DE ESTADÍSTICA (INE). Chile: Proyecciones y Estimaciones de Población 19902020. País y Regiones. Disponible en: http://www.ine.cl.

17.- BERND L, JOAN BS, MICHAEL S, BERNHARD K, LOWIE EV, LOUISA G, et al. Determinants of underdiagnosis of COPD in national and international surveys. Chest 2015; 148: 971-85.

18.- GALESANU R G, BERNARD S, MARQUIS K, LACASSE Y, POIRIER P, BOURBEAU J, et al. Obesity and chronic obstructive pulmonary disease: Is fatter really better? Can Respir J 2014; 21: 297-301.

19.- MONTES DE OCA M, TALAMO C, PÉREZ-PADILLA R, JARDIM JR, MUINO A, LÓPEZ M V, et al. Chronic obstructive pulmonary disease and body mass index in five Latin America cities: the PLATINO study. Respir Med 2008; 102: 642-50.

20.- ALVEAR T G, FIGUEROA R L P, PEÑA M C A. Perfil clínico de los pacientes ingresados al programa EPOC en un consultorio de atención primaria durante 10 años. Rev Chil Enferm Respir 2015; 31: 17-26.

21.- DIJKSTRAA E, POSTMA D S, VAN GINNEKEN B, WIELPUTZ M O, SCHMIDT M, BECKER N, et al. Novel genes for airway wall thickness identified with combined genome-wide association and expression analyses. Am J Respir Crit Care Med 2015; 191: $547-$ 56.

22.- FLORES B C, SOLÍS S M, FORTT Z A, VALDIVIA C G. Sintomatología respiratoria y enfermedad pulmonar obstructiva crónica y su asociación a contaminación intradomiciliaria en el Área Metropolitana de Santiago: Estudio Platino. Rev Chil Enferm Respir 2010; 26: 7280 .

23.- BLANC PD, MENEZES AM, PLANA E, MANNINO DM, HALLAL PC, TOREN K, et al. Occupational exposures and COPD: an ecological analysis of international data. Eur Respir J 2009; 33: 298-304.

24.- GAMONAL CONTRERAS S. El Principio De Protección Del Trabajador En La Constitución Chilena. Estudios constitucionales 2013; 11: 425-58.

25.- LÓPEZ VARELA M V, MONTES DE OCA M, HALBERT R, MUIÑO A, TÁLAMO C, PÉREZ-PADILLA $\mathrm{R}$, et al. Comorbilidades y estado de salud en individuos con y sin EPOC en 5 ciudades de América Latina: Estudio PLATINO. Arch Bronconeumol 2013; 49: 468-74.

26.- YIN H, YIN S, LIN Q, XU Y, XU H, LIU T. Prevalence of comorbidities in chronic obstructive pulmonary disease patients: A meta-analysis. Medicine (Baltimore) 2017; 96: e6836. doi: 10.1097/MD.0000000000006836.

27.- MCGARVEY L P, MAGDER S, BURKHART D, KESTEN S, LIU D, MANUEL R C, et al. Cause-specific mortality adjudication in the UPLIFT(R) COPD trial: findings and recommendations. Respir Med 2012; 106 : 515-21.

28.- MONTES DE OCA M, TALAMO C, HALBERT RJ, PÉREZ-PADILLA R, LOPEZ MV, MUINO A, et al. Frequency of self-reported COPD exacerbation and airflow obstruction in five Latin American cities: the Proyecto Latinoamericano de Investigacion en Obstruccion Pulmonar (PLATINO) study. Chest 2009; 136 : 71-8.

29.- LÓPEZ VARELA MV, MUINO A, PÉREZ PADILLA R, JARDIM JR, TALAMO C, MONTES DE OCA M, et al. [Treatment of chronic obstructive pulmonary disease in 5 Latin American cities: the PLATINO study]. Arch Bronconeumol 2008; 44: 58-64.

30.- ERNST P, SAAD N, SUISSA S. Inhaled corticosteroids in COPD: the clinical evidence. Eur Respir J 2015; 45 : 525-37.

Correspondencia a:

Dr. Jordi Olloquequi González

Instituto de Ciencias Biomédicas, Facultad de Ciencias

de la Salud, Universidad Autónoma de Chile.

Calle 5 Poniente \#1670, Edificio Aulas 3, $5^{\circ}$ Piso.

3460000 Talca, Chile.

Email: jolloquequig@uautonoma.cl 